\title{
Adaptive Adjustment of Radiation Properties for Entire Range of Axial Ratio using a Parasitic Microstrip Polarizer
}

\author{
Sungjun Yoo*, Dongeun Lee**, Gangil Byun*** and Hosung Choo ${ }^{\dagger}$
}

\begin{abstract}
This paper proposes the design of microstrip patch antennas for dual-band polarization adjustment. The antenna has a multi-layer structure for dual-band operation, and each layer contains a resonating patch with surrounding strips separated into two parts. The antenna polarization is adjusted by varying the separated positions of the strips, while fixing other design parameters. To demonstrate the feasibility, an antenna sample with right-hand circular polarization is fabricated, and its antenna characteristics are measured in a full anechoic chamber. The operating principle of polarization adjustment in the dual frequency bands is also verified by observing near electromagnetic fields and the magnetic surface current density around the antenna.
\end{abstract}

Keywords: Dual-band patch antennas, Polarization adjustment antennas, Microstrip antennas, GPS antennas

\section{Introduction}

In recent wireless communications systems, antennas are required to have multi-band characteristics with different polarization properties $[1,2]$. However, the antenna polarization is easily distorted by multipath effects, such as wave scattering, blockage, and reflection, which cause an additional power loss due to the polarization mismatch [3]. Thus, there has been a lot of effort to control the antenna polarization using various approaches, for example, cornertruncated square patches [4-7] and position-changed feeding probes $[8,9]$. However, these approaches are often limited to obtaining specific polarizations and do not provide indepth considerations on tuning the polarization properties in the entire range of the axial ratio (AR). Although reconfigurable antenna structures can adjust the antenna polarization, they have been obstructive in practical applications because of its increased design complexity and the use of additional DC bias circuit [10-12].

In this paper, we propose the design of a multi-layer patch antenna for dual-band polarization adjustment. The proposed antenna consists of two patches, each of which is composed of a resonator and surrounding strips separated into two parts. The separated positions of these strips are varied to adjust dual-band polarization properties of the antenna over the entire AR range, which includes righthand circular (RHC), left-hand circular (LHC), elliptical, and linear polarizations. The upper patch is fed by a

$\dagger$ Corresponding Author: School of Electronic and Electrical Engineering, Hongik University, Seoul, South Korea. (hschoo@hongik.ac.kr)

* School of Electronic and Electrical Engineering, Hongik University, Seoul, South Korea. (ryoonet@naver.com)

** Moasoft Co., Ltd., Seoul, South Korea. (gaiafree1@gmail.com)

*** Research Institute of Science and Technology, Hongik University, Seoul, South Korea. (kylebyun@gmail.com)

Received: November 1, 2016; Accepted: January 19, 2017 coaxial cable, and the lower patch is electromagnetic coupled to the upper patch. To demonstrate the feasibility of the dual-band polarization adjustment, we fabricate an antenna sample having the RHC polarization, and its antenna characteristics are measured in a full anechoic chamber. Then, operating principles of the proposed antenna are verified by calculating the electric and magnetic field distributions. In addition, a phase difference of the magnetic surface current density induced between the resonator and the strips is observed to compare its variation with the AR. The results prove that the proposed antenna is suitable for dual-band polarization adjustment without the use of any additional circuits.

\section{Geometry of the Proposed Antenna}

Fig. 1 shows the geometry of the proposed multi-layer patch antenna for dual-band polarization adjustment. Each layer contains a resonating patch with surrounding strips that are separated by $d_{1}$ and $d_{2}$, and their positions are adjusted by $\phi_{a r 1}$ and $\phi_{a r 2}$ to vary the polarization properties without significant frequency shifts [13]. The upper patch is fed by a coaxial cable, and its feeding positions is determined by $l_{f}$. The lower patch is then electromagnetically coupled to the upper patch through a high-dielectric CER10 substrate from Taconic $\left(\varepsilon_{\mathrm{r}}=10\right.$, $\tan \delta=0.035$ ), and their thicknesses are expressed as $h_{l}$ and $h_{2}$. The edge lengths of the two patches $\left(w_{1}\right.$ and $\left.w_{2}\right)$ are designed to be about a half wavelength $\left(\lambda_{g}\right)$ at each frequency band, and the gap distance $\left(g_{1}\right.$ and $\left.g_{2}\right)$ and strip width $\left(w_{3}\right.$ and $\left.w_{4}\right)$ are adjusted to induce strong electric fields within the gap for high magnetic surface current density. This is an important design factor for polarization adjustment because the AR of the antenna can be 


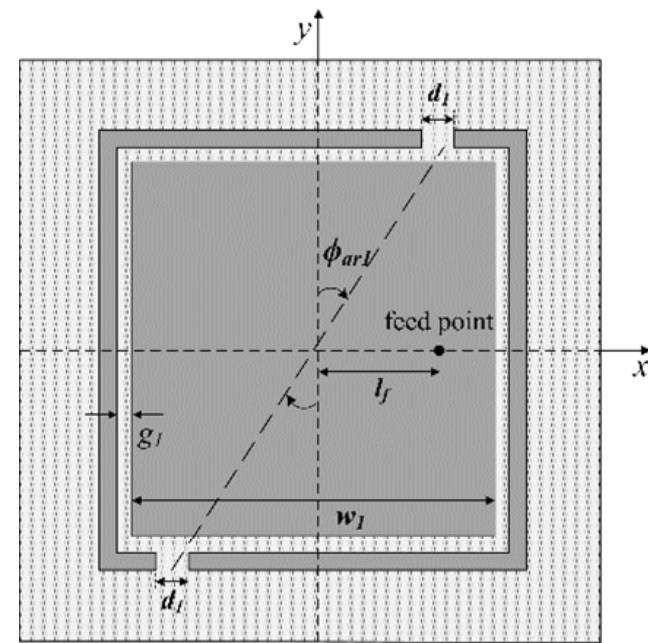

(a) Top view of upper patch

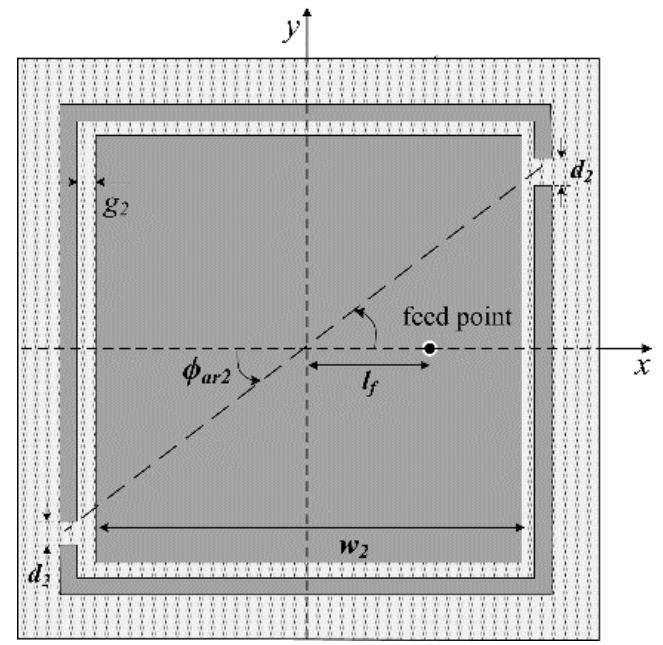

(b) Top view of lower patch

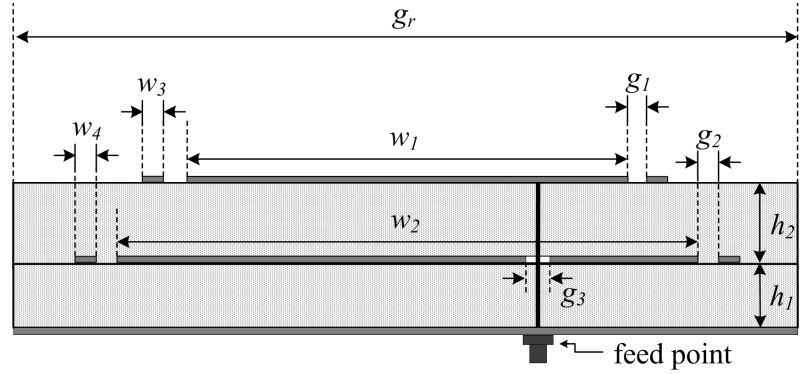

(c) Side view of the antenna

Fig. 1. Geometry of the proposed antenna

controlled by varying the phase of the magnetic surface current density, which will be analysed in Section III.

Figs. 2(a) and (b) exhibit the variations of AR values at the bore-sight direction at $1.575 \mathrm{GHz}$ and $1.227 \mathrm{GHz}$, respectively. In this observation, the values of $\phi_{a r 1}$ and $\phi_{a r 2}$ are adjusted from $-50^{\circ}$ to $50^{\circ}$ at an interval of $10^{\circ}$ while fixing those of other design parameters as specified in Table 1. At $1.575 \mathrm{GHz}$, the maximum AR value of 0.9 is
Table 1. Optimized values of the proposed antenna

\begin{tabular}{c|c}
\hline Parameters & Optimized values \\
\hline$w_{1}$ & $30.2 \mathrm{~mm}$ \\
\hline$w_{2}$ & $35.6 \mathrm{~mm}$ \\
\hline$w_{3}$ & $1.9 \mathrm{~mm}$ \\
\hline$w_{4}$ & $2.7 \mathrm{~mm}$ \\
\hline$g_{1}$ & $0.9 \mathrm{~mm}$ \\
\hline$g_{2}$ & $1.3 \mathrm{~mm}$ \\
\hline$g_{3}$ & $1.2 \mathrm{~mm}$ \\
\hline$d_{1}$ & $0.5 \mathrm{~mm}$ \\
\hline$d_{2}$ & $0.5 \mathrm{~mm}$ \\
\hline$l_{f}$ & $10.6 \mathrm{~mm}$ \\
\hline$h_{1}$ & $6.28 \mathrm{~mm}$ \\
\hline$h_{2}$ & $6.28 \mathrm{~mm}$ \\
\hline$g_{r}$ & $50 \mathrm{~mm}$ \\
\hline
\end{tabular}

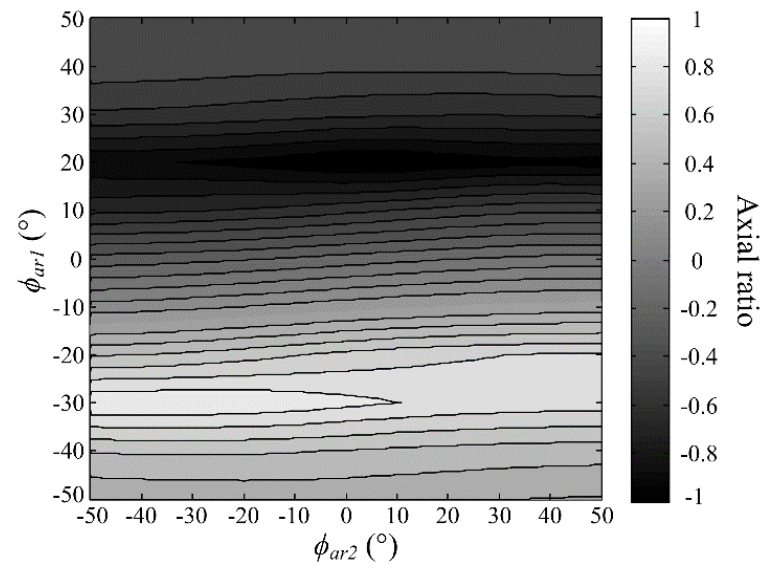

(a) Axial ratio at $1.575 \mathrm{GHz}$

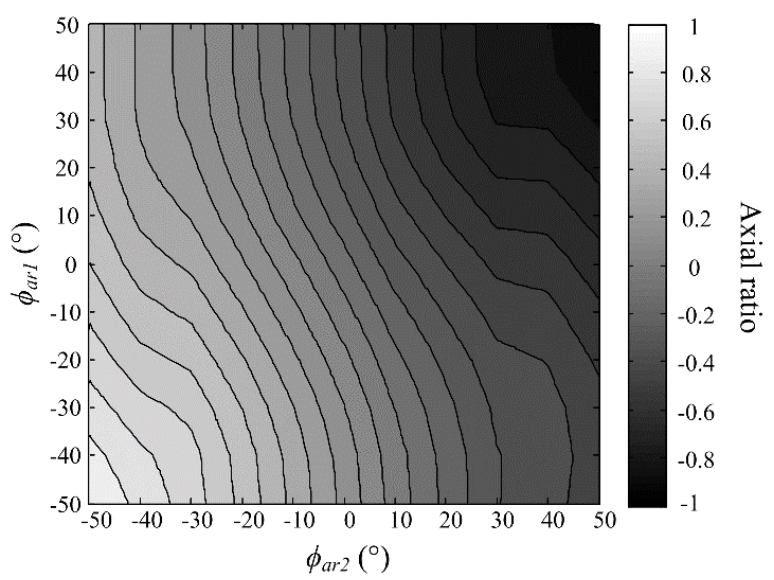

(b) Axial ratio at $1.227 \mathrm{GHz}$

Fig. 2. The values of the AR for the angle of the polarizer

observed at $\phi_{a r 1}=-30^{\circ}$ and $\phi_{a r 2}=-50^{\circ}$, and the minimum value of -0.97 is located at $\phi_{a r 1}=20^{\circ}$ and $\phi_{a r 2}=0^{\circ}$. At $1.227 \mathrm{GHz}$, the maximum value of 0.94 is found at $\phi_{\text {arl }}=$ $-50^{\circ}$ and $\phi_{a r 2}=-50^{\circ}$, and the minimum value of -0.89 is placed at $\phi_{a r 1}=50^{\circ}$ and $\phi_{a r 2}=50^{\circ}$.

The results show that we can adjust the polarization properties in the entire AR range, which includes RHC, 
LHC, linear, and elliptical polarizations. In addition, various combinations of different polarizations, e.g. linear with RHC polarizations, can also be achieved by separately varying the values of $\phi_{a r 1}$ and $\phi_{a r 2}$.

\section{Measurement and Analysis}

To demonstrate the results, we fabricated a sample antenna with the RHC polarization $\left(\phi_{\text {arl }}=-30^{\circ}, \phi_{\text {ar } 2}=\right.$ $-50^{\circ}$ ), its antenna characteristics are measured in a full anechoic chamber. Fig. 3 shows a comparison of the measured and simulated reflection coefficients as a function of frequency. The simulated values are $-11.9 \mathrm{~dB}$ at $1.575 \mathrm{GHz}$ and $-11.2 \mathrm{~dB}$ at $1.23 \mathrm{GHz}$, and the measured values are $-10.5 \mathrm{~dB}$ and $-15.4 \mathrm{~dB}$ at $1.6 \mathrm{GHz}$ and 1.25 $\mathrm{GHz}$, respectively.

The measured results are shifted by about $20 \mathrm{MHz}$ toward the upper frequency band due to fabrication error of unintended air gaps between substrates, which is not included in our EM simulation. Fig. 4 shows the measured bore-sight gain in comparison with the simulated results.

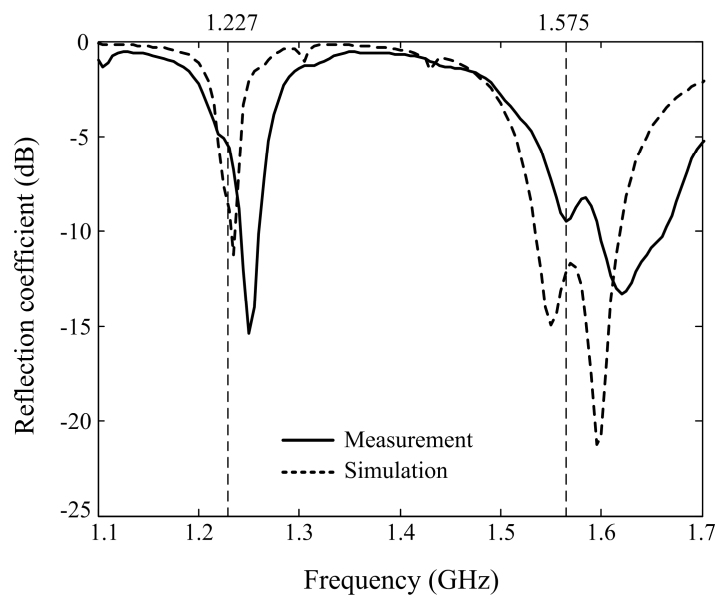

Fig. 3. Reflection coefficients of the proposed antenna

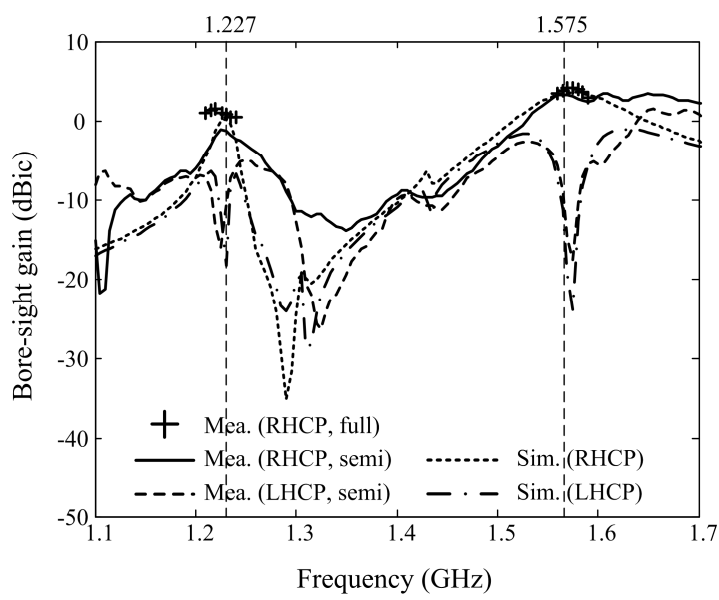

Fig. 4. Bore-sight gain of the proposed antenna
The dotted and dash-dotted lines indicate the simulated RHC and LHC polarization gains, respectively, and the measured results are specified by the solid line (RHC), dashed line (LHC), and ' + ' markers. The antenna has

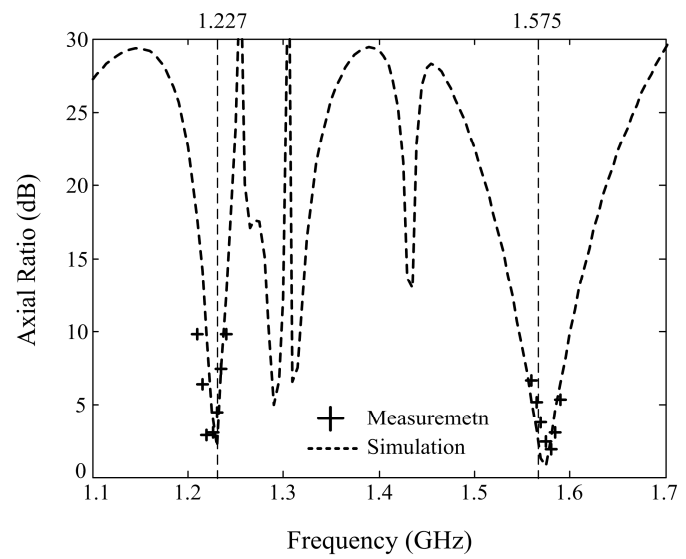

Fig. 5. Axial ratio of the proposed antenna

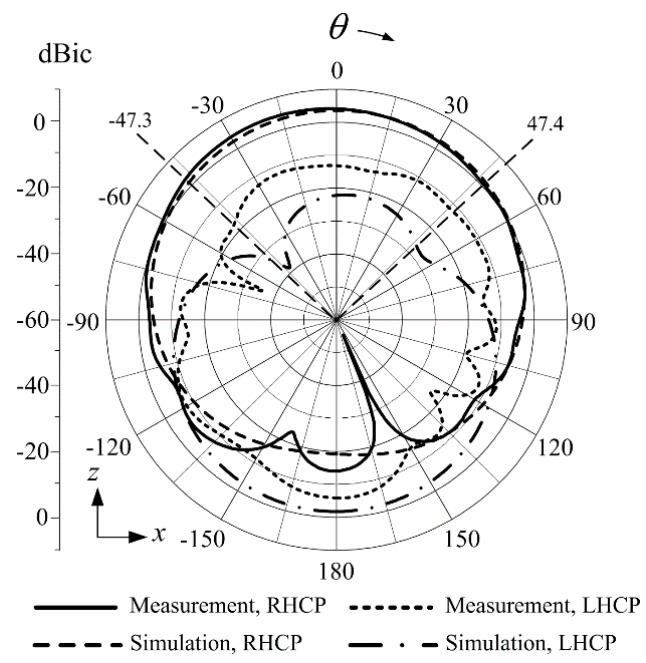

(a) $z x$-plane at $1.575 \mathrm{GHz}$

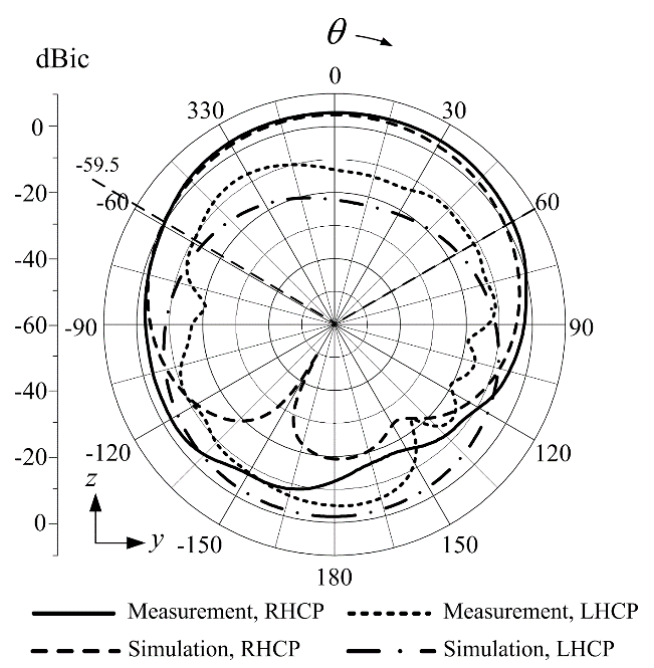

(b) zy-plane at $1.575 \mathrm{GHz}$

Fig. 6. Radiation patterns of the proposed antenna 
measured values of $4 \mathrm{dBic}$ and $0.9 \mathrm{dBic}$ at $1.575 \mathrm{GHz}$ and $1.227 \mathrm{GHz}$, respectively, and the gain in the GPS L2 band is relatively lower than the GPS L1 band due to the blockage effect in the bore-sight direction. The crosspolarization levels are $-17.2 \mathrm{~dB}(1.575 \mathrm{GHz})$ and -15.3 $\mathrm{dB}(1.227 \mathrm{GHz})$.

Fig. 5 illustrates a comparison of the AR values in the bore-sight direction. The dashed line shows the simulated results, and ' + ' markers express the measured AR values. As can be seen, the antenna is circularly polarized with the measured AR values of $1.9 \mathrm{~dB}$ and $2.8 \mathrm{~dB}$ at $1.575 \mathrm{GHz}$ and $1.227 \mathrm{GHz}$, which has a similar trend with the simulated values of $0.7 \mathrm{~dB}$ and $2.1 \mathrm{~dB}$. These AR values are increased to $10.1 \mathrm{~dB}$ and $15.5 \mathrm{~dB}$ in the GPS L1 and L2 bands when the antenna becomes elliptically polarized $\left(\phi_{\text {arl }}=-10^{\circ}, \phi_{\text {ar } 2}=30^{\circ}\right)$, which demonstrates that the polarization properties can be adjusted by simply changing the shape of the strips. Figs. 6(a) and (b) show comparisons of the measured and simulated radiation patterns in $z x$ - and

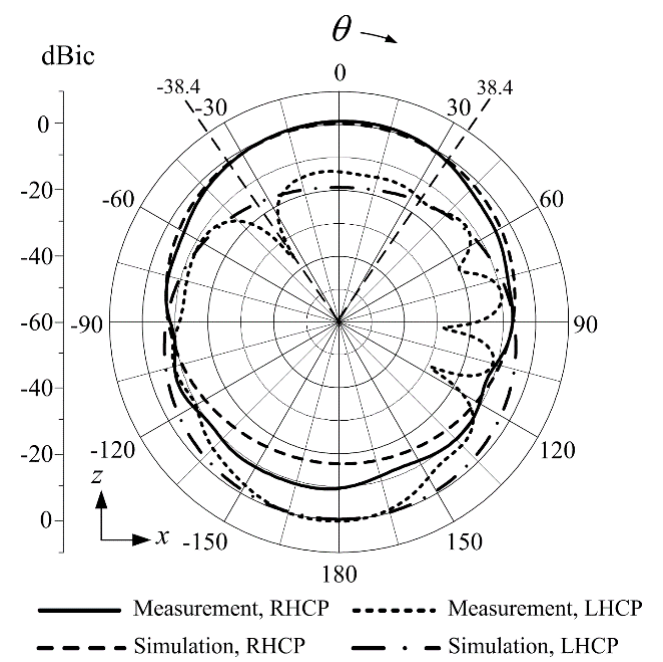

(a) $z x$-plane at $1.227 \mathrm{GHz}$

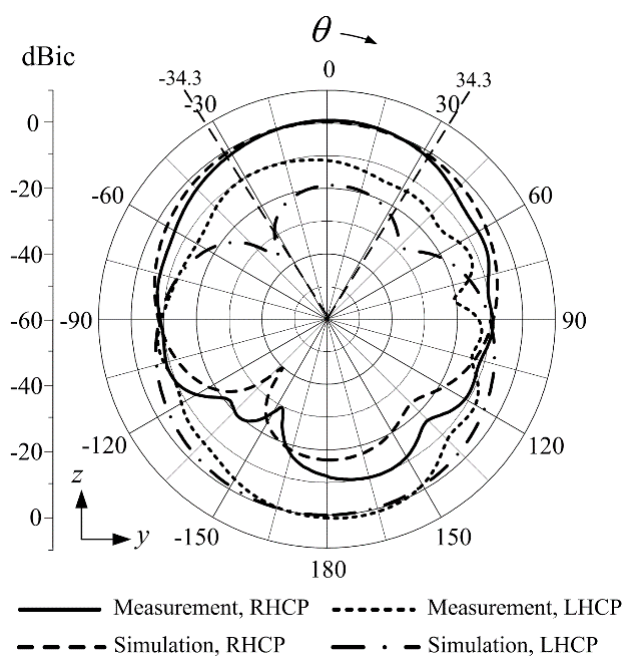

(b) zy-plane at $1.227 \mathrm{GHz}$

Fig. 7. Radiation patterns of the proposed antenna $z y$ - planes at $1.575 \mathrm{GHz}$. The half power beam widths (HPBWs) of the antenna are $94.7^{\circ}$ (zx-plane) and $119.5^{\circ}$ (zy-plane), which are similar to the simulated value of $110^{\circ}$ (zx- and $z y$-planes). Figs. 7(a) and (b) illustrate patterns at $1.227 \mathrm{GHz}$, and their HPBWs are $76.8^{\circ}$ and $68.6^{\circ}$ in $z x$ and $z y$-planes, respectively. We can verify that the fabricated antenna has circular polarization in the upper hemisphere with average cross-polarization levels of -14.7 $\mathrm{dB}(1.575 \mathrm{GHz})$ and $-10.7 \mathrm{~dB}(1.227 \mathrm{GHz})$. The height difference $h_{2}$ also varies the AR properties; however, this $\mathrm{AR}$ variation is caused by an undesired frequency shift as shown in Fig. 8(a). On the other hand, $\phi_{\text {arl }}$ allows for the adjustment of polarization properties in entire AR range from -1 to 1 without a significant frequency shift of less than $9 \mathrm{MHz}$, as presented in Fig. 8(b). Figs. 9(a) and (b) illustrate magnetic field distributions that are observed at $61 \times 61$ points on a cross section with a dimension of 60 $\mathrm{mm} \times 27 \mathrm{~mm}$, when input power is $1 \mathrm{~W}$. As can be seen, the $\mathrm{H}$-field is confined between the upper and lower patches with an average field strength of $15.1 \mathrm{~A} / \mathrm{m}$ at 1.575 $\mathrm{GHz}$, and a strong field is distributed between the lower patch and the ground with an average value of $24.5 \mathrm{~A} / \mathrm{m}$ at $1.227 \mathrm{GHz}$.

Figs. 10(a) and (b) show top views of the E-field

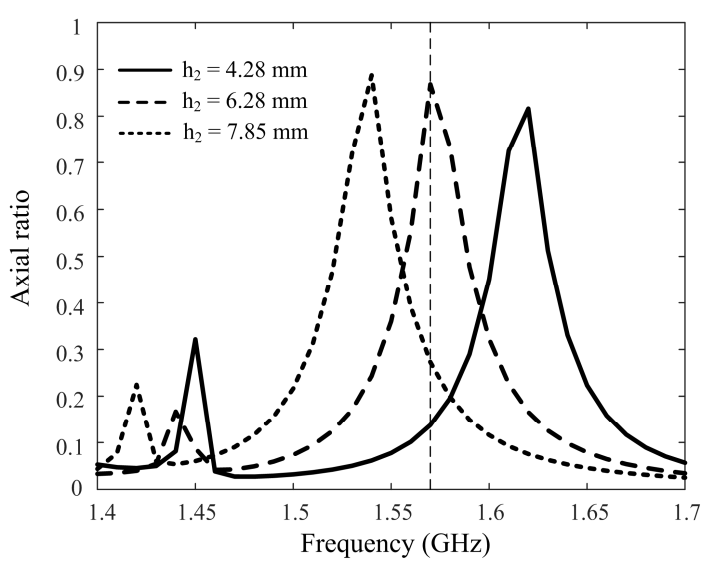

(a) Variation of AR according to $h_{2}$

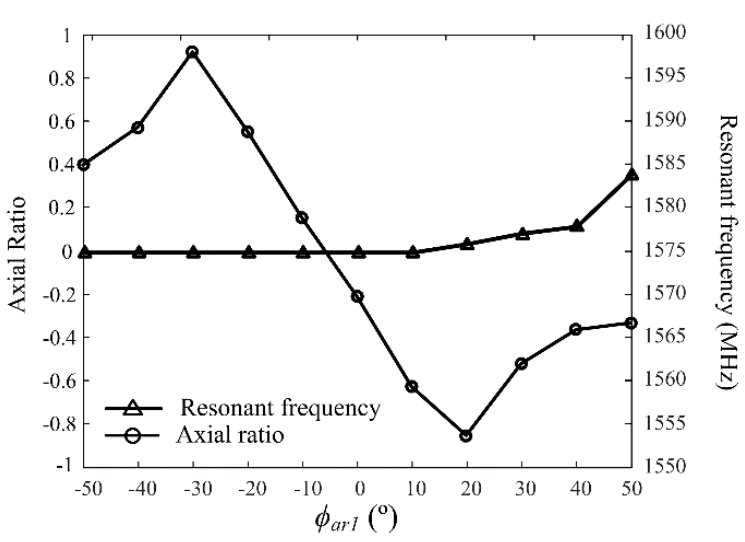

(b) AR and resonant frequency properties

Fig. 8. Variation of axial ratio 
distributions at the upper and lower patches at $1.575 \mathrm{GHz}$ and $1.227 \mathrm{GHz}$, respectively. As expected, strong electric fields are confined between the patch and the strips, which implies that high magnetic surface current density equivalently exists within the surface area of the gap. Since the shape of the parasitic strips affects the phase of the magnetic surface current density with little change in magnitude, only the polarization properties are adjusted without a significant frequency shift.

Figs. 11(a) and (b) show comparisons between the AR and the phase difference of the magnetic surface current density at $1.575 \mathrm{GHz}$ and $1.227 \mathrm{GHz}$, respectively. We

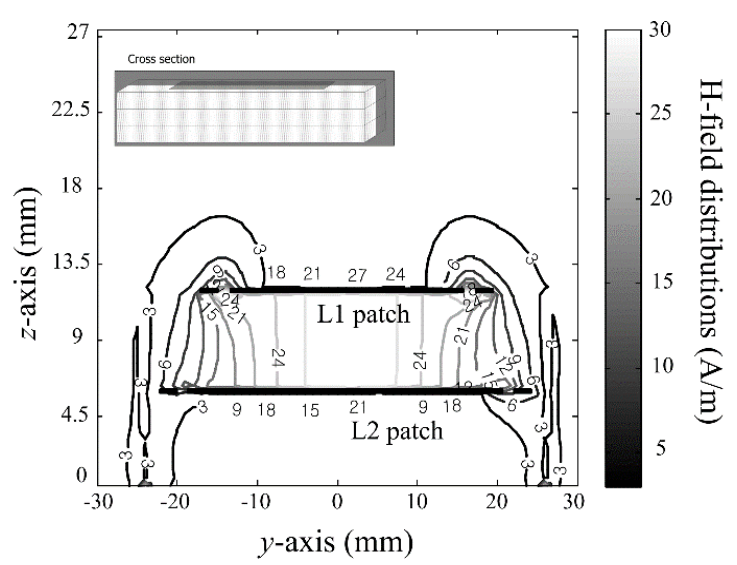

(a) $1.575 \mathrm{GHz}$

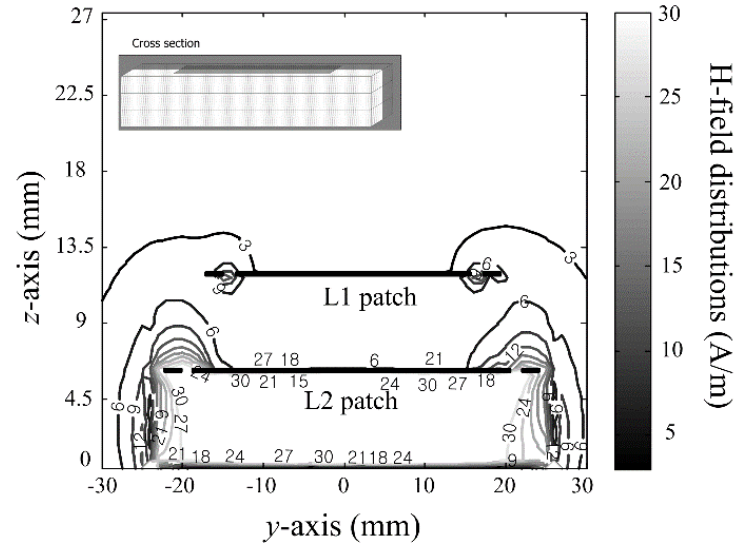

(b) $1.227 \mathrm{GHz}$

Fig. 9. H-field distribution of the proposed antenna

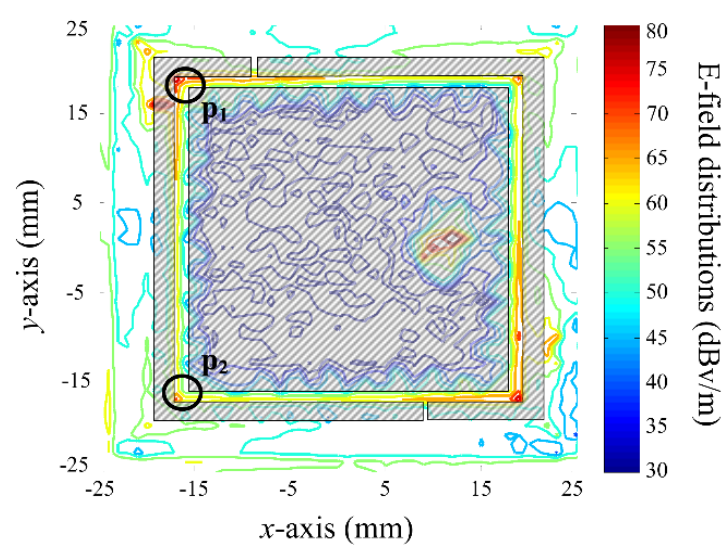

(a) $1.575 \mathrm{GHz}$

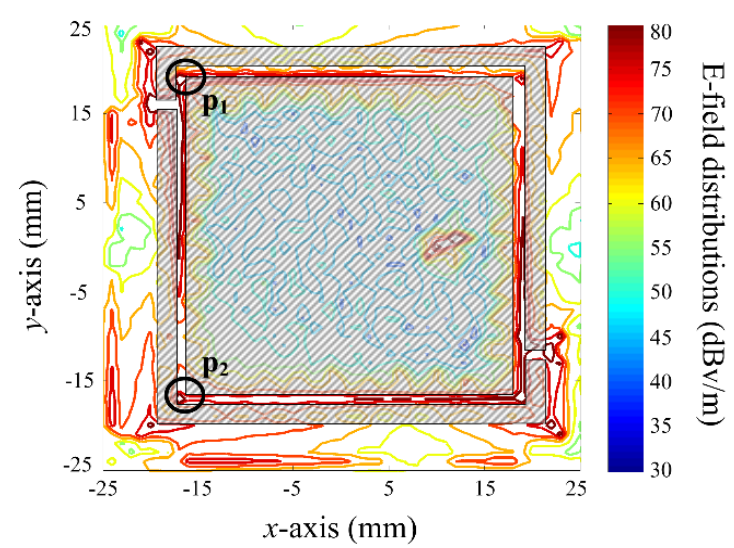

(b) $1.227 \mathrm{GHz}$

Fig. 10. E-field distribution of the proposed antenna

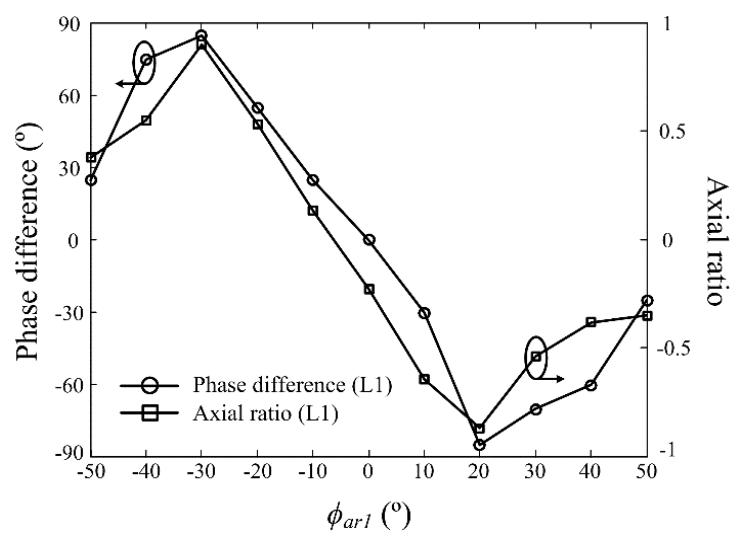

(a) $1.575 \mathrm{GHz}$

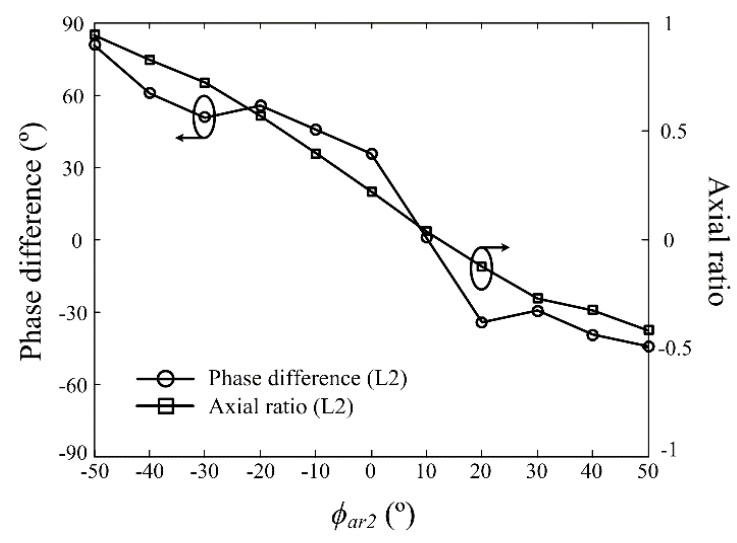

(b) $1.227 \mathrm{GHz}$

Fig. 11. Phase of magnetic surface current density and AR 
calculated the phase difference using phase values at two points of $p_{1}$ and $p_{2}$, as specified in Fig. 10. The square and circular markers indicate the AR and the phase difference, respectively, and the angles of $\phi_{a r 1}$ and $\phi_{a r 2}$ are varied from $-50^{\circ}$ to $50^{\circ}$, while fixing other parameters as listed in Table 1. If we assume the center of the patch as ' $O$ ', the angle between $\overline{O p_{1}}$ and $\overline{O p_{2}}$ is $90^{\circ}$, geometrically. Thus, when the antenna has the right-hand circular polarization with $\mathrm{AR}=0.9$, the phase at $\mathrm{p}_{1}$ should lead the phase at $\mathrm{p}_{2}$ by $85^{\circ}$, while a $85^{\circ}$ lag is needed for $\mathrm{AR}=-0.97$. Zero phase difference is observed for the linear polarization. In our approach, we intentionally adjusted the phase difference between $p_{1}$ and $p_{2}$ from $-90^{\circ}$ to $90^{\circ}$ by varying $\phi_{a r l}$ and $\phi_{a r 2}$ to achieve the AR in the entire range from -1 to 1 .

\section{Conclusion}

We have investigated the design of a multi-layer patch antenna for dual-band polarization adjustment. The proposed antenna consisted of two resonating patches with parasitic strips, and the antenna polarization is adjusted by rotating the separated positions of the strips. The feasibility of the polarization adjustment was verified by measuring antenna characteristics of the sample antenna with the RHC polarization. The measured bore-sight gains were 4 $\mathrm{dBic}$ and $0.9 \mathrm{dBic}$ with the cross-polarization levels of $-17.2 \mathrm{~dB}$ and $-15.3 \mathrm{~dB}$ at $1.575 \mathrm{GHz}$ and $1.227 \mathrm{GHz}$, respectively. The strong $\mathrm{H}$-fields were confined between patches with average field strengths of $15.1 \mathrm{~A} / \mathrm{m}$ at 1.575 $\mathrm{GHz}$ and $24.5 \mathrm{~A} / \mathrm{m}$ at $1.227 \mathrm{GHz}$. We also verified that the phase difference has a similar trend with the AR, and the circular polarization can be achieved when the amplitude of the phase difference is close to $90^{\circ}$. The result demonstrated that the proposed antenna was suitable to adjust polarization properties in dual bands.

\section{Acknowledgements}

This work was supported by the research fund of Signal Intelligence Research Center supervised by Defense Acquisition Program Administration and Agency for Defense Development of Korea.

\section{References}

[1] V. Erceg, H. Sampath, and S. Catreux-Erceg, "Dualpolarization versus single-polarization MIMO channel measurement results and modelling," IEEE Trans. Wireless Commun., vol. 5, no. 1, pp. 28-33, January 2006.

[2] D. Sarkar, K. V Srivastava, and K. Saurav, "A compact microstrip-fed triple band-notched UWB monopole antenna," IEEE Antennas Wirel. Propag. Lett., vol. 13, pp. 396-399, February 2014.

[3] D. C. Cox, "Antenna diversity performance in mitigating the effects of portable radiotelephone orientation and multipath propagation," IEEE Trans. Wireless Commun., vol. 31, no. 5, pp. 620-628, May 1983.

[4] C. Shichai, L. Guangcong, L. Tingfen, L. Xiangguo, and D. Zhiqi, "Compact dual-band GPS microstrip antenna using multilayer LTCC substrate," IEEE Antennas Wirel. Propag. Lett., vol. 9, pp. 421-423, May 2010.

[5] S. A. Bokhari, J. -F. Zurcher, J. R. Mosig, and F. E. Gardiol, "A small microstrip patch antenna with a convenient tuning option," IEEE Trans. Antennas Propag., vol. 44, no. 11, pp. 1521-1528, November 1996.

[6] T.-N. Chang, J.-M. Lin, "Circularly polarized antenna having two linked slot-rings," IEEE Trans. Antennas Propag., vol. 59, no. 8, pp. 3057-3060, June 2011.

[7] G. Byun, J. Hyun, S. M. Seo, and H. Choo, "Optimum array configuration to improve null steering time for mobile CRPA Systems," Journal of Electromagnetic Engineering and Science, vol. 16, no. 2, pp. 74-79, April 2016.

[8] H.-M. Chen, K.-L. Wong. "On the circular polarization operation of annular-ring microstrip patch antennas," IEEE Trans. Antennas Propag., vol. 47, no. 8, pp. 1289-1292, August 1999.

[9] G Byun, S Kim, H. Choo, "Design of a dual-band GPS antenna using a coupled feeding structure for high isolation in a small array," Microwave Opt. Technol. Lett., vol.56, no. 2, pp. 359-361, February 2014.

[10] B. Li, Q. Xue, "Polarization-Reconfigurable omnidirectional antenna combining dipole and loop radiators," IEEE Antennas Wirel. Propag. Lett., vol. 12, pp. 1102-1105, September 2013.

[11] C. Sim, Y. Liao, H. Lin, "Polarization reconfigurable eccentric annular ring slot antenna design," IEEE Trans. Antennas Propag., vol. 63, no. 9, pp. 41524155, September 2015.

[12] J. -S. Row, W. -L. Liu, T-. R. Chen, "Circular polarization and polarization reconfigurable designs for annular slot antennas," IEEE Trans. Antennas Propag., vol.60, no. 12, pp. 5998-6002, December 2012.

[13] G. Byun and H. Choo, "Antenna polarisation adjustment for microstrip patch antennas using parasitic elements," Electron. Lett., vol. 51, no. 14, pp. 1046-1048, July 2015. 


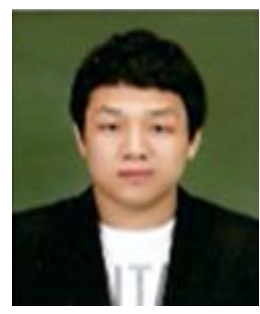

Sungjun Yoo was born in Seoul, Korea, in 1989. He received the B.S. and M.S. degrees in electronic and electrical engineering from Hongik University, Seoul, Korea, in 2014 and 2016, respectively. He is currently working toward the Ph.D. degree in electronics and com-puter engineering at Hongik University, Seoul, Korea. His research interests include the global positioning system antennas, antenna arrays, and position optimization of array elements for adaptive beamforming

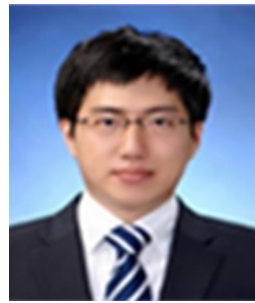

Dongeun Lee was born in Seoul, Korea, in 1986. He received the B.S. and M.S. degrees in electronic and electrical engineering from Hongik University, Seoul, Korea, in 2013 and 2015, respectively. $\mathrm{He}$ is currently working in Moasoft Co., Ltd., Seoul, South Korea. His research interests include the global positioning system antennas, antenna arrays, and position optimization of aircraft antennas.

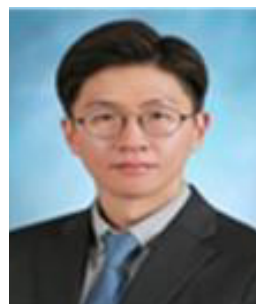

Gangil Byun (S'12, M'15) received his B.S. and M.S. degrees in electronic and electrical engineering from Hongik University, Seoul, Korea in 2010 and 2012, respectively, and his Ph.D. degree in electronics and computer engineering from Hanyang University, Seoul, Korea in 2015. He is currently a research professor in the Research Institute of Science and Technology at Hongik University. Dr. Byun's principal areas of research are in circularly-polarized antenna design, vehicular and aeronautic antenna design, global positioning system antenna design, antenna and array configuration optimization, and array antenna design for various adaptive beamforming applications such as direction of arrival estimation, interference mitigation, and radar. He has actively contributed to the consideration of both antenna characteristics and signal processing perspectives for the improvement of overall beamforming performances. He has published 27 journal papers, 58 conference papers, 11 patents to date and received the Young Scientist Award from the International Union of Radio Science in 2016 and several Best Paper Awards from the Korean Institute of Electromagnetic Engineering and Science.

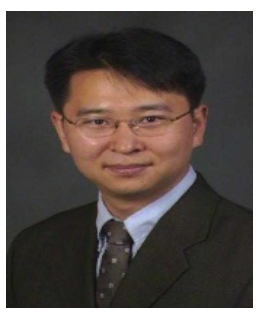

Hosung Choo (S'00, M'04, SM'11) was born in Seoul, Korea, in 1972 . He received the B.S. degree in radio science and engineering from Hanyang University in Seoul in 1998, and the M.S. and Ph.D. degrees in electrical and computer engineering from the University of Texas at Austin, in 2000 and 2003, respectively. In September 2003, he joined the school of electronic and electrical engineering, Hongik University, Seoul, Korea, where he is currently a full professor. His principal areas of re-search are the use of the optimization algorithm in developing antennas and microwave absorbers. His studies include the design of small antennas for wireless communications, reader and tag antennas for RFID, and on-glass and conformal antennas for vehicles and aircraft. 